\title{
Upregulated matrix metalloproteinase-2 and downregulated tissue factor pathway inhibitor-2 are risk factors for lymph node metastasis and perineural invasion in pancreatic carcinoma
}

\author{
This article was published in the following Dove Press journal: \\ OncoTargets and Therapy \\ 7 October 2015 \\ Number of times this article has been viewed
}

\author{
Lu-Lu Zhai ${ }^{1,2, *}$ \\ Yang Wu ${ }^{1,2, *}$ \\ Chong-Yang Cai, \\ Zhi-Gang Tang ',2 \\ 'Department of General Surgery, \\ Affiliated Provincial Hospital, \\ Anhui Medical University, ${ }^{2}$ Anhui \\ Province Key Laboratory of \\ Hepatopancreatobiliary Surgery, Hefei, \\ People's Republic of China \\ *These authors contributed equally \\ to this work
}

\begin{abstract}
Background: Dysregulated expression of matrix metalloproteinase (MMP)-2 and tissue factor pathway inhibitor (TFPI)-2 is closely associated with tumorigenesis and tumor progression. The aim of this work was to determine the predictive values of MMP-2 and TFPI-2 in identifying lymph node metastasis (LNM) and perineural invasion (PNI) in pancreatic carcinoma.
\end{abstract}

Methods: Formalin-fixed and paraffin-embedded tissue samples containing pancreatic carcinoma tissues and their corresponding para-carcinoma tissues were obtained from 122 patients with pancreatic carcinoma. The expression levels of MMP-2 and TFPI-2 were evaluated by immunohistochemistry. The roles of MMP-2 and TFPI-2 in predicting LNM and PNI in pancreatic carcinoma were analyzed.

Results: The level of MMP-2 expression was markedly increased in pancreatic carcinoma tissues (76.9\%) compared with para-carcinoma tissues $(29.2 \% ; P<0.05)$. In contrast, there was obviously decreased TFPI-2 expression level in pancreatic carcinoma tissues (29.2\%) compared with para-carcinoma tissues $(77.7 \% ; P<0.001)$. Additionally, MMP-2 expression was significantly positively correlated with LNM $(r=0.468, P<0.01)$ and PNI $(r=0.637, P<0.01)$. In contrast, TFPI-2 expression was strongly negatively correlated with LNM $(r=-0.396, P<0.001)$ and PNI $(r=-0.460, P<0.001)$. Logistic regression analysis showed that high MMP-2 expression and low TFPI-2 expression acted as independent predictors for LNM and PNI in pancreatic carcinoma.

Conclusion: Taken together, our findings suggest that upregulated MMP-2 and downregulated TFPI-2 serve as useful predictors for a high risk of LNM and PNI. Obtaining information on the expression of MMP-2 and TFPI-2 before surgery may predict the occurrence of LNM and PNI, thereby permitting reasonable and effective surgical treatment for patients with pancreatic carcinoma.

Keywords: MMP-2, TFPI-2, immunohistochemistry

\section{Introduction}

Pancreatic carcinoma is an aggressive and rapidly fatal malignant disease which is the ninth most common malignancy and the fourth leading cause of carcinoma-related deaths, with an average 5-year survival rate of less than 5\% in the USA. ${ }^{1,2}$ Currently, early diagnosis and radical surgery provide the only chance of long-term survival for patients with pancreatic carcinoma. Unfortunately, most patients have already presented with serious local invasion and/or distant metastases when pancreatic carcinoma is first discovered, thereby missing the optimal timing for curative surgical resection. ${ }^{3}$ Although great efforts have been made in primary prevention, early diagnosis, and
Correspondence: Zhi-Gang Tang Anhui Province Key Laboratory of Hepatopancreatobiliary Surgery, 17 Lujiang Road, Hefei 23000 I, Anhui Province, People's Republic of China

Tel +86 I38 66II 2279

Fax +86 55। 62283280

Email tzg567@I63.com 
clinical treatment of pancreatic carcinoma, the 5-year survival rate remains unsatisfactory for all stages and races. ${ }^{4}$ Therefore, in order to improve the prognosis of the patients, there is an urgent need to increase our understanding of the evolution of this deadly disease.

The biological behavior of pancreatic carcinoma is that tumor cells disseminate via adjacent tissues, peripheral nerves, lymphatic vessels, and blood vessels to implement local infiltration, perineural invasion (PNI), lymphatic metastasis, and distant metastasis; these events depend on special tumor microenvironmental factors to accelerate the degradation of the extracellular matrix (ECM). ${ }^{5}$ The ECM is an important physiological barrier in tumor invasion and metastasis which, by interacting with tumor cells, can block them from entering the adjacent tissues. The integrity of the ECM is regulated by the balance between matrix metalloproteinases (MMPs) and their inhibitors; some previous studies have indicated that an imbalance between MMPs and their inhibitors can enhance the degradation of the ECM, thereby promoting the infiltration and migration of tumor cells. ${ }^{6-8}$ MMP-2 is an important member of the MMP family which plays a significant part in the development of some neoplastic diseases, and a high level of MMP-2 expression in pancreatic carcinoma tissues has been previously elucidated. ${ }^{9-12}$ Tissue factor pathway inhibitor (TFPI)-2 is a Kunitz-type serine proteinase repressor which is widely produced in a variety of normal human tissues and organs, including the pancreas. TFPI-2 can inhibit the activity of various proteases, including MMPs. ${ }^{13}$ Therefore, TFPI-2 is considered a protector that prevents components of the ECM from degrading, thereby combating the infiltration and migration of tumor cells. ${ }^{14-17}$ Previous studies have suggested that TFPI-2 expression is reduced in all kinds of human solid tumors, including pancreatic carcinoma. ${ }^{18-20}$

Lymph node metastasis (LNM) and PNI are two important aggressive biologic and clinicopathologic characteristics of pancreatic carcinoma and are closely related to the unfavorable outcome of patients after surgery. ${ }^{21-24}$ In general, the patients with LNM or PNI should undergo more extensive lymphadenectomy or more aggressive neural dissection around the vessels. However, preoperatively, it is difficult to determine whether or not LNM or PNI are present. Despite several molecular markers having been proposed to predict LNM and PNI in pancreatic carcinoma, the potential predictive values of the molecular markers involved have had no significant effect in pancreatic carcinoma. A previous study has suggested that MMP-2 protein expression is elevated and TFPI-2 protein expression decreased in pancreatic carcinoma tissues; the differential expressions of the proteins show a negative correlation and may have opposite effects on the progression of disease. ${ }^{25}$ In addition, high MMP-2 expression and low TFPI-2 expression are significantly associated with a poor prognosis in patients with pancreatic carcinoma. ${ }^{25}$ However, it is unclear whether MMP-2 and TFPI-2 can be used as significant predictive biomarkers for predicting LNM and PNI in pancreatic carcinoma. Therefore, in this work, we performed an immunohistochemical study to validate the levels of MMP-2 and TFPI-2 expression in pancreatic carcinoma tissues, and to investigate the roles of MMP-2 and TFPI-2 in predicting LNM and PNI in pancreatic carcinoma.

\section{Materials and methods Patients and tissue samples}

The study was conducted in agreement with the Declaration of Helsinki and was approved by the Human Scientific Ethics Committee of Anhui Medical University (Hefei, People's Republic of China). Pancreatic carcinoma tissues and para-carcinoma tissues (defined as pancreatic tissue from $2 \mathrm{~cm}$ beyond the tumor margin) were collected from a total of 122 patients who underwent curative surgical resection (tumorectomy plus lymphadenectomy) and who were pathologically diagnosed with pancreatic carcinoma between 2008 and 2011 at the Affiliated Provincial Hospital of Anhui Medical University (Hefei, People's Republic of China). All postoperative samples were fixed in $10 \%$ formaldehyde, embedded in paraffin wax, and stored at the specimen bank of the pathology department. All patients signed the informed consent and had not received any anticancer therapy before surgery.

All patients had complete clinical and pathological data, including age ( $<60$ or $\geq 60$ years), sex (male or female), tumor diameter ( $<20$ or $\geq 20 \mathrm{~mm}$ ), tumor location (head or body/tail), preoperative serum carbohydrate antigen 19-9 (CA19-9) concentrations ( $\leq 37$ or $>37 \mathrm{U} / \mathrm{mL}$ ), tumor differentiation (well or moderate/poor), LNM (absent or present), and PNI (absent or present). These patients consisted of 65 men and 57 women, with a mean age of 56 years (range: 46-66 years). LNM and PNI were observed directly by postoperative pathological examination. Tumor differentiation was defined according to the grading systems of the National Cancer Institute (NCI). ${ }^{26}$

\section{Immunohistochemical staining and scoring for MMP-2 and TFPI-2}

To detect the expression of MMP-2 and TFPI-2 in pancreatic tissues, immunohistochemical staining was performed using 
a two-step protocol according to the manufacturer's instructions. As previously described, tissue sections ( $4 \mu \mathrm{m}$ thick) from paraffin-embedded specimens were heated at $60^{\circ} \mathrm{C}$ for 20 minutes, dewaxed with xylene, rehydrated through gradient ethanol (100\%, 95\%, and 75\%, respectively), and washed with phosphate-buffered saline (PBS). ${ }^{25}$ Antigen retrieval was then performed by applying a microwave-heating procedure in $0.01 \mathrm{~mol} / \mathrm{L}$ sodium citrate buffer $(\mathrm{pH} 6.0)$ at $80^{\circ} \mathrm{C}$ for 20 minutes. After endogenous peroxidase activity was blocked with $0.3 \% \mathrm{H}_{2} \mathrm{O}_{2}$ for 10 minutes, the sections were incubated at $4{ }^{\circ} \mathrm{C}$ overnight with polyclonal primary antibodies (antiMMP-2; Santa Cruz Biotechnology Inc., Dallas, TX, USA; anti-TFPI-2; Zhongshan Golden Bridge Biotechnology Co., Ltd., Beijing, People's Republic of China) in a humid chamber. After washing with PBS, the sections were incubated with biotinylated secondary mouse antibody for 30 minutes at a constant $37^{\circ} \mathrm{C}$ and then with horseradish peroxidase-labeled streptavidin for 30 minutes at a constant $37^{\circ} \mathrm{C}$. After further washing with PBS, chromogenic immunolocalization was implemented using 3,3-diaminobenzidine (Sigma-Aldrich Co., St Louis, MO, USA) substrate. Finally, the sections were counterstained with hematoxylin before dehydration and mounting. In addition, the negative controls were arranged by replacing the primary antibodies with PBS under the same conditions.

The semiquantitative assessment of immunostaining was performed according to the percentage and intensity of stained cells, as previously described..$^{25}$ The percentage of stained cells was graded as follows: 0 points, no staining; 1 point $<10 \%$; 2 points, $10 \%-30 \%$; 3 points $>30 \%$ of stained cells. The intensity of staining was: 0 points, absent; 1 point, weak; 2 points, moderate; 3 points, strong. The immunoreactive total score was the product of the points for the intensity and percentage of staining. A final total score $\leq 3$ was defined as low expression, and $>3$ was determined to be high expression. All immunostained slides were reviewed independently by two experienced pathologists who were blinded to each other's results. For all differences, a consensus was reached by joint evaluation.

\section{Statistical analysis}

Statistically significant differences between tumor tissues and the surrounding nontumor tissues were evaluated using the Pearson's chi-squared test, based on the final total immunostaining scores. The relationships between LNM, PNI, and other clinicopathological data were also analyzed using Pearson's chi-squared test. The associations of LNM and PNI with MMP-2 expression and TFPI-2 expression were analyzed using either Pearson's chi-squared test or Spearman's rank correlation test. Logistic regression analysis was applied to determine the independent predictors for the risk of LNM and PNI. All statistical analyses were performed using the software package SPSS version 17.0 (SPSS, Inc., Chicago, IL, USA). All analyses were determined by a two-tailed test, and $P<0.05$ was considered statistically significant.

\section{Results \\ Correlation between LNM, PNI, and other clinicopathologic data}

The relationships between LNM, PNI, and other clinicopathological data were analyzed. As shown in Table 1, the data demonstrated that LNM was associated with tumor diameter $(P=0.026)$ and preoperative serum CA19-9 concentrations $(P=0.013)$. However, there was no obvious association between LNM and age, sex, tumor location, or tumor differentiation $(P>0.05)$. A weak association was also found between PNI and preoperative serum CA19-9 concentrations $(P=0.042)$, but there was no significant association between PNI and age, sex, tumor diameter, tumor location, or tumor differentiation $(P>0.05)$.

\section{Immunohistochemical characteristics of MMP-2 and TFPI-2 in pancreatic carcinoma tissues and para-carcinoma tissues}

The results of staining showed that MMP-2 and TFPI-2 were mainly localized to the cytoplasm of cells (Figure 1). Our results showed that MMP-2 expression was higher in pancreatic carcinoma tissues $(100 / 130,76.9 \%)$ than in para-carcinoma tissues $(38 / 130,29.2 \%)$. In contrast, TFPI-2 expression was lower in pancreatic carcinoma tissues $(38 / 130,29.2 \%)$ than in para-carcinoma tissues $(101 / 130$, $77.7 \%$ ). Statistical analysis showed that there were significant statistical differences in the expression levels of MMP-2 $(P=0.001)$ and TFPI-2 $(P<0.001)$ between pancreatic carcinoma tissues and para-carcinoma tissues (Table 2).

\section{Correlation of LNM with MMP-2 and TFPI-2 staining}

The relationships of LNM with MMP-2 and TFPI-2 staining were evaluated. The results demonstrated that there was a significant positive association between MMP-2 expression and LNM ( $r=0.468, P<0.001)$. In contrast, there was a significant negative association between TFPI-2 expression and LNM in pancreatic carcinoma $(r=-0.396, P<0.001)$ (Table 3). 
Table I Relationships between the other clinicopathological parameters and the status of LNM and PNI

\begin{tabular}{|c|c|c|c|c|c|c|c|}
\hline \multirow[t]{2}{*}{ Parameters } & \multirow[t]{2}{*}{ Number } & \multicolumn{3}{|l|}{ LNM } & \multicolumn{3}{|l|}{ PNI } \\
\hline & & Absent & Present & $P$-value & Absent & Present & $P$-value \\
\hline \multicolumn{8}{|l|}{ Age (years) } \\
\hline$<60$ & 61 & 24 & 37 & 0.846 & 21 & 40 & 0.627 \\
\hline$\geq 60$ & 69 & 26 & 43 & & 21 & 48 & \\
\hline \multicolumn{8}{|l|}{ Sex } \\
\hline Male & 69 & 32 & 37 & 0.070 & 22 & 47 & 0.913 \\
\hline Female & 61 & 18 & 43 & & 20 & 41 & \\
\hline \multicolumn{8}{|l|}{ Tumor diameter } \\
\hline$<20 \mathrm{~mm}$ & 62 & 30 & 32 & 0.026 & 23 & 39 & 0.265 \\
\hline$\geq 20 \mathrm{~mm}$ & 68 & 20 & 48 & & 19 & 49 & \\
\hline \multicolumn{8}{|l|}{ Tumor location } \\
\hline Head & 64 & 25 & 39 & 0.890 & 21 & 43 & 0.904 \\
\hline Body/tail & 66 & 25 & 41 & & 21 & 45 & \\
\hline \multicolumn{8}{|l|}{ Serum CAI9-9 } \\
\hline$\leq 37 \mathrm{U} / \mathrm{mL}$ & 43 & 23 & 20 & 0.013 & 19 & 24 & 0.042 \\
\hline$>37 \mathrm{U} / \mathrm{mL}$ & 87 & 27 & 60 & & 23 & 64 & \\
\hline \multicolumn{8}{|l|}{ Differentiation } \\
\hline Moderate/poor & 74 & 28 & 46 & 0.867 & 24 & 50 & 0.972 \\
\hline Well & 56 & 22 & 34 & & 18 & 38 & \\
\hline
\end{tabular}

Abbreviations: CA19-9, carbohydrate antigen 19-9; LNM, lymph node metastasis; PNI, perineural invasion.
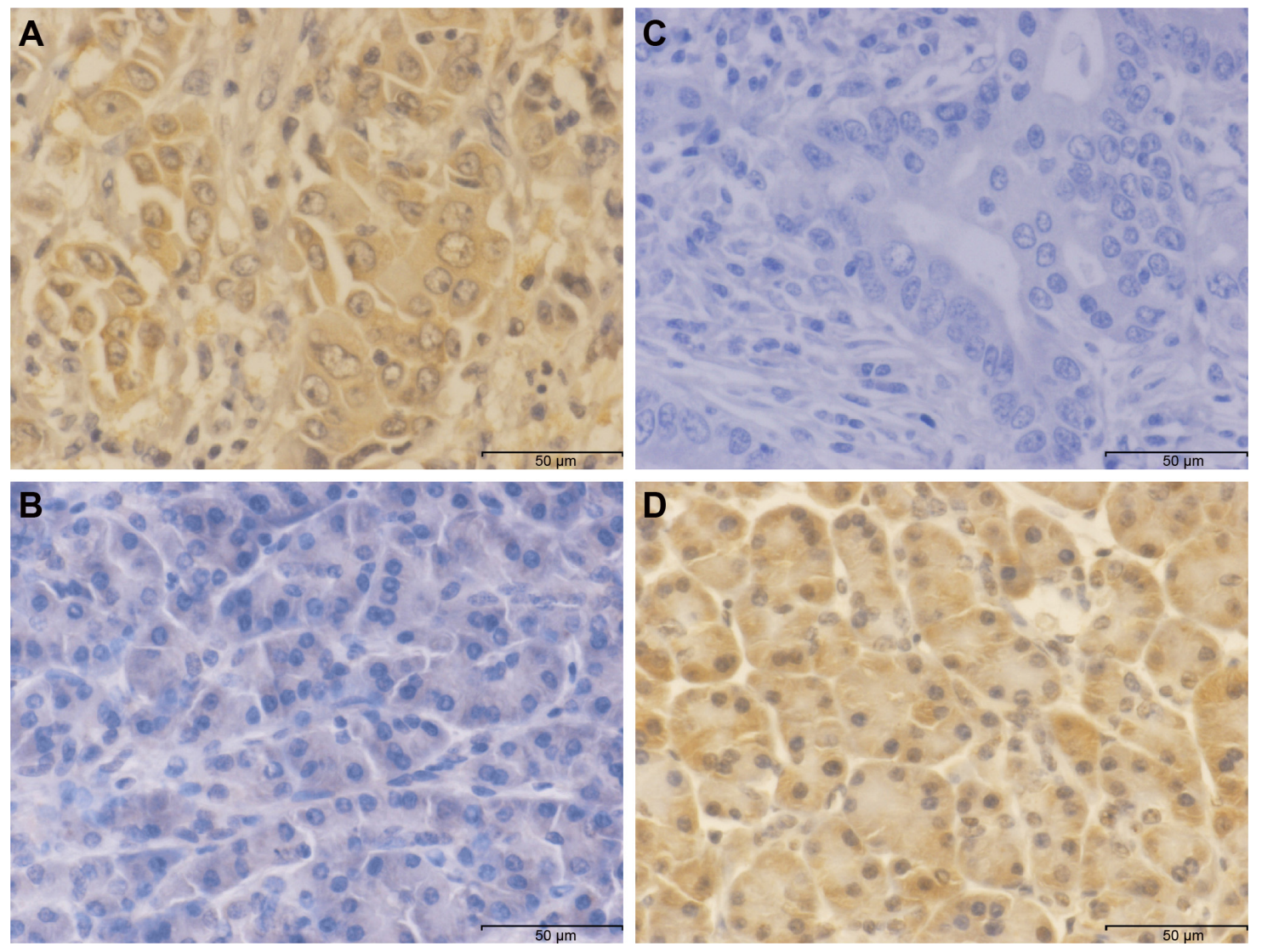

Figure I Immunohistochemistry of MMP-2 and TFPI-2 in pancreatic carcinoma tissues and para-carcinoma tissues.

Notes: The MMP-2 and TFPI-2 are mainly focused on the cytoplasm of cells. (A) Shows the high-level expression of MMP-2 in pancreatic carcinoma tissues. (B) Shows the low-level expression of MMP-2 in para-carcinoma tissues. (C) Shows the low-level expression of TFPI-2 in pancreatic carcinoma tissues. (D) Shows the high-level expression of TFPI-2 in para-carcinoma tissues. All images were taken at $400 \times$ magnification.

Abbreviations: MMP, matrix metalloproteinase; TFPI, tissue factor pathway inhibitor. 
Table 2 Differential expression of MMP-2 and TFPI-2 in pancreatic carcinoma tissues and corresponding para-carcinoma tissues $(n=130)$

\begin{tabular}{|c|c|c|c|c|c|c|}
\hline \multirow[t]{2}{*}{ Tissues } & \multicolumn{3}{|c|}{ MMP-2 expression } & \multicolumn{3}{|c|}{ TFPI-2 expression } \\
\hline & Low (\%) & High (\%) & $P$-value & Low (\%) & High (\%) & P-value \\
\hline Carcinoma tissues & $30(23.1)$ & $100(76.9)$ & 0.001 & $92(70.8)$ & $38(29.2)$ & $<0.001$ \\
\hline Para-carcinoma tissues & $92(70.8)$ & $38(29.2)$ & & $29(22.3)$ & I0I (77.7) & \\
\hline
\end{tabular}

Abbreviations: MMP, matrix metalloproteinase; TFPI, tissue factor pathway inhibitor.

\section{Correlation of PNI with MMP-2 and TFPI-2 staining}

As shown in Table 3, the results of the evaluation showed that the level of MMP-2 expression in pancreatic carcinoma tissues was significantly and positively correlated with PNI $(r=0.637, P<0.001)$. In contrast, TFPI-2 expression was significantly and negatively correlated with PNI in pancreatic carcinoma $(r=-0.460, P<0.001)$.

\section{Multivariate analysis for independent risk factors of LNM and PNI}

In order to identify which variable was the independent predictor for predicting the risk of LNM and PNI, a logistic regression analysis was performed. This showed that high MMP-2 expression served as an independent risk factor for LNM (hazard ratio $[\mathrm{HR}]=7.587 ; 95 \%$ confidence interval $[\mathrm{CI}] 2.657-21.665 ; P<0.001)$ and $\mathrm{PNI}(\mathrm{HR}=24.127 ; 95 \% \mathrm{CI}$ $7.068-82.358 ; P<0.001)$ in pancreatic carcinoma. Additionally, multivariate analysis showed that low TFPI-2 expression also acted as an independent risk factor for $\mathrm{LNM}(\mathrm{HR}=0.271$; 95\% CI 0.109-0.676; $P=0.005)$ and PNI $(\mathrm{HR}=0.199 ; 95 \% \mathrm{CI}$ $0.071-0.561 ; P=0.002)$ in pancreatic carcinoma (Table 4).

\section{Discussion}

Identifying which specific molecular biomarkers to use to predict the occurrence of LNM and PNI could make a significant contribution to improving the prognosis for patients with pancreatic carcinoma. In the present study, we detected the levels of MMP-2 and TFPI-2 expression in pancreatic carcinoma tissues and para-carcinoma tissues by immunohistochemistry, and investigated the roles of MMP-2 and TFPI-2 in predicting LNM and PNI in pancreatic carcinoma. Our results showed that MMP-2 expression was significantly elevated in pancreatic carcinoma tissues relative to that in para-carcinoma tissues; these results were consistent with previous findings that MMP-2 expression was upregulated in pancreatic carcinoma tissues. ${ }^{9-12,25}$ We also demonstrated that TFPI-2 expression was significantly reduced in pancreatic carcinoma tissues compared to para-carcinoma tissues, thereby verifying the previous findings that TFPI-2 expression was remarkably downregulated in pancreatic carcinoma tissues. ${ }^{18-20,25}$ Interestingly, these results suggested that the level of MMP-2 expression had a significantly positive correlation with LNM and PNI, whereas the level of TFPI-2 expression was significantly negatively correlated with LNM and PNI in pancreatic carcinoma. Furthermore, high MMP-2 expression and low TFPI-2 expression in pancreatic carcinoma tissues acted as independent risk factors for LNM and PNI. Therefore, our findings show that MMP-2 and TFPI-2 both play an important role in the development of LNM and PNI in pancreatic carcinoma.

There is increasing evidence to indicate that MMP-2 plays a prominent role in LNM and progression of tumor. ${ }^{27-30}$ Mönig et $\mathrm{al}^{27}$ suggested that MMP-2 staining is strongly correlated with LNM and tumor progression in gastric carcinoma, which may be a useful indicator of tumor progression, especially for LNM. Wu et $\mathrm{al}^{28}$ reported that the level of intratumoral MMP-2 protein staining is significantly elevated and might

Table 3 Associations between the expression of MMP-2 and TFPI-2 and the status of LNM and PNI

\begin{tabular}{|c|c|c|c|c|c|c|c|c|}
\hline \multirow[t]{2}{*}{ Variables } & \multicolumn{4}{|l|}{ LNM } & \multicolumn{4}{|l|}{ PNI } \\
\hline & Absent & Present & $r$-value & $P$-value & Absent & Present & $r$-value & $P$-value \\
\hline \multicolumn{9}{|c|}{ MMP-2 expression } \\
\hline Low & 24 & 6 & 0.468 & $<0.001$ & 26 & 4 & 0.637 & $<0.001$ \\
\hline High & 26 & 74 & & & 16 & 84 & & \\
\hline \multicolumn{9}{|c|}{ TFPI-2 expression } \\
\hline Low & 24 & 68 & -0.396 & $<0.001$ & 17 & 75 & -0.460 & $<0.001$ \\
\hline High & 26 & 12 & & & 25 & 13 & & \\
\hline
\end{tabular}

Note: Data presented as number of patients.

Abbreviations: MMP, matrix metalloproteinase; LNM, lymph node metastasis; PNI, perineural invasion; TFPI, tissue factor pathway inhibitor. 
Table 4 Logistic regression analysis of factors associated with LNM and PNI

\begin{tabular}{|c|c|c|c|c|c|c|}
\hline \multirow[t]{2}{*}{ Variables } & \multicolumn{3}{|l|}{ LNM } & \multicolumn{3}{|l|}{ PNI } \\
\hline & HR & $95 \% \mathrm{Cl}$ & $P$-value & HR & $95 \% \mathrm{Cl}$ & $P$-value \\
\hline $\begin{array}{l}\text { MMP-2 expression } \\
\text { (low vs high) }\end{array}$ & 7.587 & $2.657-21.665$ & $<0.001$ & 24.127 & $7.068-82.358$ & $<0.001$ \\
\hline $\begin{array}{l}\text { TFPI-2 expression } \\
\text { (low vs high) }\end{array}$ & 0.271 & $0.109-0.676$ & 0.005 & 0.199 & $0.07 \mid-0.56 I$ & 0.002 \\
\hline
\end{tabular}

Abbreviations: $\mathrm{Cl}$, confidence interval; HR, hazard ratio; LNM, lymph node metastasis; MMP, matrix metalloproteinase; PNI, perineural invasion; TFPI, tissue factor pathway inhibitor.

be a useful marker for predicting the risk of cervical LNM in patients with papillary thyroid carcinoma. Huang et $\mathrm{al}^{29}$ showed that the positive rate of MMP-2 expression is higher in lymph-node-metastatic nasopharyngeal carcinoma than in chronic nasopharyngitis and nonmetastatic nasopharyngeal carcinoma, and that upregulated MMP-2 may enhance the metastatic capability of carcinoma cells through the overexpression of ECM metalloproteinase inducer. Xiang et a ${ }^{30}$ indicated that high-level expression of MMP-2 protein is an independent risk factor for developing LNM; the combination of intratumoral hypoxia-inducible factor- $1 \alpha$, vascular endothelial growth factor, and MMP-2 staining may also be useful as a molecular prediction model for LNM in hepatocellular carcinoma. Our data showed that overexpressed MMP-2 has a dramatic and positive association with LNM, serving as an independent predictor of LNM in pancreatic carcinoma. Furthermore, high MMP-2 expression was significantly and positively associated with PNI, acting as an independent prognosticator of PNI in pancreatic carcinoma. Our results combined with previous findings suggest that MMP-2 may play a facilitative role in the development of LNM and PNI in pancreatic carcinoma. Nevertheless, for some other types of human tumor, high-level expression of MMP-2 shows no correlation with the risk of LNM..$^{31,32}$ For instance, Krecicki et $\mathrm{a}^{31}$ reported that there is no significant correlation between strong MMP-2 immunohistochemical staining and the risk of LNM in patients with laryngeal cancer. Moreover, Campos et $\mathrm{al}^{32}$ demonstrated that MMP-2 immunoreactivity is not associated with a greater risk of LNM in invasive penile carcinoma. Therefore, more studies are required to support our findings.

With respect to the relationship between TFPI-2 expression and LNM in malignant tumors, Zhang et $\mathrm{al}^{33}$ suggested that TFPI-2 expression has a decreasing trend and is significantly correlated with LNM in the progression of cervical cancer. Xu et $\mathrm{al}^{34}$ indicated that negative or low TFPI-2 expression is significantly associated with LNM, vessel invasion, poor histologic grade, tumor progression, tumor recurrence, and poor outcome, and is a potential prognostic tool in breast cancer. In addition, Hibi et al ${ }^{35-37}$ detected methylation of the TFPI-2 gene by quantitative methylation-specific polymerase chain reaction and found that TFPI-2 gene methylation is common in the serum of patients with colorectal cancer and those with gastric cancer, and that gene methylation is significantly related to LNM and distant metastases. Aberrant methylation of the TFPI-2 gene is regarded as significant in the reduction of TFPI- 2 expression in human cancers. ${ }^{38}$ In the current study, our findings showed that the downregulation of TFPI-2 expression was markedly negatively correlated with LNM and acted as an independent factor for high risk of LNM in pancreatic carcinoma. In addition, low TFPI-2 expression was significantly and negatively associated with PNI, acting as an independent predictive factor for the risk of PNI in pancreatic carcinoma. These findings reveal that TFPI-2 may have an inhibiting effect on the development of LNM and PNI in pancreatic carcinoma.

A major limitation of this single-center work was the relatively small sample size. In addition, it is inevitable that there will be a selection bias in any retrospective study. More prospective studies with a larger cohort of subjects are therefore needed to support our findings.

Taken together, the current findings have confirmed the high-level expression of MMP-2 and the low-level expression of TFPI- 2 in pancreatic carcinoma tissues. Upregulated MMP-2 and downregulated TFPI-2 are significantly correlated with LNM and PNI, and serve as independent predictors for the risk of LNM and PNI in pancreatic carcinoma. Therefore, combined detection of MMP- 2 and TFPI- 2 may be considered a potentially and promising tool to predict LNM and PNI in pancreatic carcinoma. Our present findings could make a momentous contribution to decisions on the patterns of surgical treatment if information on MMP-2 and TFPI-2 expression can be obtained preoperatively. Currently, only a few reports are available regarding the roles of MMP-2 and TFPI-2 in identifying LNM and PNI in pancreatic carcinoma. Consequently, further studies will be necessary to ascertain whether MMP-2 and TFPI-2 are useful predictive factors for LNM and PNI in pancreatic carcinoma. 


\section{Acknowledgments}

The authors thank Yu-Xia Zhang for polishing the manuscript. The authors thank all the anonymous reviewers for their helpful suggestions on the quality improvement of our paper. This study was supported by the National Natural Science Foundation of China (number 81272740).

\section{Disclosure}

The authors report no conflicts of interest in this work.

\section{References}

1. Siegel R, Ma J, Zou Z, Jemal A. Cancer statistics, 2014. CA Cancer J Clin. 2014;64:9-29.

2. Gong ZH, Holly EA, Bracci PM. Survival in population-based pancreatic cancer patients: San Francisco Bay area, 1995-1999. Am J Epidemiol. 2011;174:1373-1381.

3. Niederhuber JE, Brennan MF, Menck HR. The National Cancer Data Base report on pancreatic cancer. Cancer. 1995;76:1671-1677.

4. Vincent A, Herman J, Schulick R, Hruban RH, Goggins M. Pancreatic cancer. Lancet. 2011;378:607-620.

5. Binker MG, Binker-Cosen MJ, Binker-Cosen AA, Cosen-Binker LI Microenvironmental factors and extracellular matrix degradation in pancreatic cancer. JOP. 2014;15:280-285.

6. Ellenrieder V, Adler G, Gress TM. Invasion and metastasis in pancreatic cancer. Ann Oncol. 1999;10 Suppl 4:46-50.

7. Hirata M, Sato T, Tsumagari M, Hashizume K, Ito A. Discoordinate regulation of expression of matrix metalloproteinases and tissue inhibitor of metalloproteinases-3 in bovine endometrial stromal cells on type-I collagen gel. Biol Pharm Bull. 2003;26:1013-1017.

8. Miyagi Y, Yasumitsu H, Eki T, et al. Assignment of the human PP5/ TFPI-2 gene to 7q22 by FISH and PCR-based human/rodent cell hybrid mapping panel analysis. Genomics. 1996;35:267-268.

9. Iki K, Tsutsumi M, Kido A, et al. Expression of matrix metalloproteinase 2 (MMP-2), membrane-type 1 MMP and tissue inhibitor of metalloproteinase 2 and activation of proMMP-2 in pancreatic duct adenocarcinomas in hamsters treated with $\mathrm{N}$-nitrosobis(2-oxopropyl) amine. Carcinogenesis. 1999;20:1323-1329.

10. Määttä M, Soini Y, Liakka A, Autio-Harmainen H. Differential expression of matrix metalloproteinase (MMP)-2, MMP-9, and membrane type 1-MMP in hepatocellular and pancreatic adenocarcinoma: implications for tumor progression and clinical prognosis. Clin Cancer Res. 2000;6:2726-2734.

11. Ellenrieder V, Alber B, Lacher U, et al. Role of MT-MMPs and MMP-2 in pancreatic cancer progression. Int J Cancer. 2000;85:14-20.

12. Roy R, Zurakowski D, Wischhusen J, et al. Urinary TIMP-1 and MMP-2 levels detect the presence of pancreatic malignancies. Br J Cancer. 2014;111:1772-1779.

13. Herman MP, Sukhova GK, Kisiel W, et al. Tissue factor pathway inhibitor-2 is a novel inhibitor of matrix metalloproteinases with implications for atherosclerosis. J Clin Invest. 2001;107:1117-1126.

14. Rao CN, Reddy P, Liu Y, et al. Extracellular matrix-associated serine protease inhibitors ( $\mathrm{Mr} 33,000,31,000$, and 27,000) are single-gene products with differential glycosylation: cDNA cloning of the 33-kDa inhibitor reveals its identity to tissue factor pathway inhibitor-2. Arch Biochem Biophys. 1996;335:82-92.

15. Rao CN, Cook B, Liu Y, et al. HT-1080 fibrosarcoma cell matrix degradation and invasion are inhibited by the matrix-associated serine protease inhibitor TFPI-2/33 kDa MSPI. Int J Cancer. 1998;76: 749-756.

16. Izumi $\mathrm{H}$, Takahashi $\mathrm{C}, \mathrm{Oh} \mathrm{J}$, Noda $\mathrm{M}$. Tissue factor pathway inhibitor-2 suppresses the production of active matrix metalloproteinase- 2 and is down-regulated in cells harboring activated ras oncogenes. FEBS Lett. 2000;481:31-36.
17. Konduri SD, Rao CN, Chandrasekar N, et al. A novel function of tissue factor pathway inhibitor-2 (TFPI-2) in human glioma invasion. Oncogene. 2001;20:6938-6945.

18. Jiang P, Watanabe H, Okada G, et al. Diagnostic utility of aberrant methylation of tissue factor pathway inhibitor 2 in pure pancreatic juice for pancreatic carcinoma. Cancer Sci. 2006;97:1267-1273.

19. Tang Z, Geng G, Huang Q, et al. Prognostic significance of tissue factor pathway inhibitor-2 in pancreatic carcinoma and its effect on tumor invasion and metastasis. Med Oncol. 2010;27:867-875.

20. Tang Z, Geng G, Huang Q, et al. Expression of tissue factor pathway inhibitor 2 in human pancreatic carcinoma and its effect on tumor growth, invasion, and migration in vitro and in vivo. J Surg Res. 2011;167:62-69.

21. Ozaki H, Hiraoka T, Mizumoto R, et al. The prognostic significance of lymph node metastasis and intrapancreatic perineural invasion in pancreatic cancer after curative resection. Surg Today. 1999;29:16-22.

22. Nakagohri T, Kinoshita T, Konishi M, Takahashi S, Gotohda N. Nodal involvement is strongest predictor of poor survival in patients with invasive adenocarcinoma of the head of the pancreas. Hepatogastroenterology. 2006;53:447-451.

23. Buc E, Couvelard A, Kwiatkowski F, et al. Adenocarcinoma of the pancreas: Does prognosis depend on mode of lymph node invasion? Eur J Surg Oncol. 2014;40:1578-1585.

24. Bapat AA, Hostetter G, Von HoffDD, Han H. Perineural invasion and associated pain in pancreatic cancer. Nat Rev Cancer. 2011;11:695-707.

25. Zhai LL, Cai CY, Wu Y, Tang ZG. Correlation and prognostic significance of MMP-2 and TFPI-2 differential expression in pancreatic carcinoma. Int J Clin Exp Pathol. 2015;8:682-691.

26. National Cancer Institute [webpage on the Internet]. Tumor Grade; 2013. Available from: http://www.cancer.gov/about-cancer/diagnosisstaging/prognosis/tumor-grade-fact-sheet. Accessed September 9, 2015.

27. Mönig SP, Baldus SE, Hennecken JK, et al. Expression of MMP-2 is associated with progression and lymph node metastasis of gastric carcinoma. Histopathology. 2001;39:597-602.

28. Wu G, Zhou Y, Li T, Guo J, Zhou Z. Immunohistochemical levels of matrix metalloproteinase- 2 and $C D 44$ variant 6 protein in the diagnosis and lateral cervical lymph node metastasis of papillary thyroid carcinoma. J Int Med Res. 2013;41:816-824.

29. Huang T, Chen MH, Wu MY, Wu XY. Correlation between expression of extracellular matrix metalloproteinase inducer and matrix metalloproteinase-2 and cervical lymph node metastasis of nasopharyngeal carcinoma. Ann Otol Rhinol Laryngol. 2013;122:210-215.

30. Xiang ZL, Zeng ZC, Fan J, Tang ZY, Zeng HY, Gao DM. Gene expression profiling of fixed tissues identified hypoxia-inducible factor- $1 \alpha$, VEGF, and matrix metalloproteinase- 2 as biomarkers of lymph node metastasis in hepatocellular carcinoma. Clin Cancer Res. 2011;17:5463-5472.

31. Krecicki T, Zalesska-Krecicka M, Jelen M, Szkudlarek T, Horobiowska M. Expression of type IV collagen and matrix metalloproteinase-2 (type IV collagenase) in relation to nodal status in laryngeal cancer. Clin Otolaryngol Allied Sci. 2001;26:469-472.

32. Campos RS, Lopes A, Guimarães GC, Carvalho AL, Soares FA. E-cadherin, MMP-2, and MMP-9 as prognostic markers in penile cancer: analysis of 125 patients. Urology. 2006;67:797-802.

33. Zhang Q, Zhang Y, Wang SZ, et al. Reduced expression of tissue factor pathway inhibitor-2 contributes to apoptosis and angiogenesis in cervical cancer. J Exp Clin Cancer Res. 2012;31:1.

34. XuC, Wang $\mathrm{H}, \mathrm{HeH}$, et al. Low expression of TFPI-2 associated with poor survival outcome in patients with breast cancer. BMC Cancer. 2013;13:118.

35. Hibi K, Goto T, Kitamura YH, et al. Methylation of TFPI2 gene is frequently detected in advanced well-differentiated colorectal cancer. Anticancer Res. 2010;30:1205-1207.

36. Hibi K, Goto T, Shirahata A, et al. Detection of TFPI2 methylation in the serum of colorectal cancer patients. Cancer Lett. 2011;311:96-100.

37. Hibi K, Goto T, Shirahata A, et al. Detection of TFPI2 methylation in the serum of gastric cancer patients. Anticancer Res. 2011;31:3835-3838

38. Sierko E, Wojtukiewicz MZ, Kisiel W. The role of tissue factor pathway inhibitor-2 in cancer biology. Semin Thromb Hemost. 2007;33:653-659. 


\section{Publish your work in this journal}

OncoTargets and Therapy is an international, peer-reviewed, open access journal focusing on the pathological basis of all cancers, potential targets for therapy and treatment protocols employed to improve the management of cancer patients. The journal also focuses on the impact of management programs and new therapeutic agents and protocols on

patient perspectives such as quality of life, adherence and satisfaction. The manuscript management system is completely online and includes a very quick and fair peer-review system, which is all easy to use. Visit http://www.dovepress.com/testimonials.php to read real quotes from published authors.

Submit your manuscript here: http://www.dovepress.com/oncotargets-and-therapy-journal 